\title{
Surface Porosity During Vacuum Bag-Only Prepreg Processing: Causes and Mitigation
}

\section{Strategies}

Lee Hamill*, Timotei Centea, Steven Nutt

Department of Chemical Engineering and Materials Science, University of Southern California, Los Angeles, CA 90089, USA

*Corresponding Author, Tel: +1 512913 7151. Email: 1hamill@ usc.edu

\begin{abstract}
In this study, we employ a parametric approach coupled with surface analysis to identify the source(s) of surface porosity and to develop effective mitigation strategies. Results confirmed that surface porosity was primarily associated with air that was trapped at the tool-prepreg interface during layup. The magnitude and distribution of surface porosity was affected by multiple parameters, including vacuum hold time, freezer and out time, and material and process modifications that affect air evacuation. The results indicated that prepreg out time (and thus tack) and vacuum quality were the primary drivers of surface porosity; for example, surface porosity decreased by $83 \%$ after just four days of out time and by $99 \%$ after 14 days of out time. These factors were used to formulate guidelines to mitigate surface porosity by (a) increasing the driving force for air evacuation and/or (b) increasing the permeability of the tool-prepreg interface.
\end{abstract}

Keywords: Prepreg (A), Porosity (B), Surface Analysis (D); Out-of-autoclave

\section{Introduction}

In recent years, the manufacturing of fiber-reinforced polymer (FRP) composites has shifted from autoclave processing toward out-of-autoclave (OoA) approaches [1]. Autoclave processing is robust, utilizing above-ambient pressures during high temperature cure to suppress 
the formation of defects, particularly voids. However, the high capital and operating costs, long cycle times, and process flow restrictions motivate the demand for more cost-effective and flexible alternatives. Vacuum bag only (VBO) prepreg processing is one such approach. VBO prepregs are vacuum bag-cured in conventional ovens, and are therefore compacted only by an atmospheric pressure differential of $101.3 \mathrm{kPa}(1 \mathrm{~atm}$, compared to $~ 5$ atm typical in autoclaves). To achieve autoclave quality levels under this reduced processing pressure, VBO prepregs feature a partially impregnated microstructure (by design) that allows the evacuation of a majority of the air extant between and within the laminate plies. This distinctive characteristic, along with several bagging arrangements and cure cycle modifications, allows the manufacture of defect-free parts with high microstructural quality. Nevertheless, the lack of positive pressure during processing renders laminates cured through VBO methods more susceptible to certain defect-causing phenomena.

Surface porosity often arises on the tool-side surface of composite laminates made from VBO prepregs. While it is generally not detrimental to mechanical properties, surface porosity degrades the aesthetic quality of the part and must often be remedied, incurring additional time and cost. Several solutions to eliminate it currently exist. For example, during layup, a resin-rich surfacing ply can be added between the first prepreg ply and the tool plate to produce a smooth, resin-rich surface. However, this adds parasitic weight to the final product and may not be viable in weight-critical applications. Post-cure operations include gel coating and painting, which also add weight to the final product as well as time and cost to the manufacturing process. The ability to consistently produce void-free surfaces without unnecessary materials or process steps, while much needed, is presently unavailable. 
Surface porosity has been addressed in previous studies, but the primary causes are not comprehensively understood, and few truly effective mitigation strategies have been proposed, particularly for non-autoclave processes. Herring et al. reported that when using a non-autoclave process based on bladder-induced consolidation (the Quickstep method), a decrease in compaction pressure resulted in an increase in surface porosity, indicating that resin pressure is an influential process parameter and pointing to why surface porosity arises more often in nonautoclave than in autoclave processes [2]. Darrow et al. used a design of experiments (DoE) approach to identify sources of surface porosity in autoclave-processed parts [3]. Several factors were considered including prepreg supplier, moisture on the tool surface, and loss of vacuum during autoclave staging. None of the parameters were identified as primary sources of surface porosity, but the authors inadvertently discovered that use of a release film at the tool-part interface resulted in a void-free surface. This solution works well for flat laminates and is commonly used for such applications [4,5], but is not viable for contoured parts due to the difficulty of draping a polymer film over tooling with multiple curvatures.

Recent studies have suggested that surface porosity results from air that becomes trapped between the prepreg and tool plate during layup [6-8]. In a previous study, we reported that composite laminates made from woven prepreg exhibit more surface porosity than laminates made from unidirectional (UD) prepreg, an observation attributed to the initial morphology of the prepreg [6]. Air entrapment is less likely with UD prepregs because of the more uniform surface topography. In the same study, we investigated the effect of tool surface roughness on surface porosity to determine if a rougher tool plate created a more permeable tool-part interface, but results showed no identifiable trend. 
Grigoreiv et al. attempted to reduce surface porosity during VBO processing by manipulating other tool plate properties. First, they studied the effect of tool surface energy on surface porosity by treating tool plates with atmospheric pressure plasma [7]. The results did not indicate a relationship between surface energy and surface porosity, but tool plates with higher surface roughness resulted in laminates with reduced surface porosity. Then, the same group investigated the effect of tool topography on surface porosity [8]. Different microstructural patterns were created on the tool surface, confirming that surface topography, microstructural spacing, and material cure cycle have an effect on the resulting surface porosity quantity. While our study showed no relationship between tool roughness and surface porosity, this study suggested that surface porosity can be reduced by optimizing the shape and size of microstructures present on the tool surface.

A review of the literature indicates that surface porosity may be governed by both prepreg material properties and tool properties, but does not clarify the relative importance of each factor. The literature also contains numerous studies focused on the nucleation, growth and migration of bulk porosity $[9,10]$, as well as on the material and processing factors governing internal voids [11]. However, none of these works consider surface defects. In addition, few studies have directly considered the role of other process parameters, including pressure and room temperature vacuum hold time, on such surface defects. The mitigation strategies that have been proposed thus far are either only viable in specific cases (e.g. the use of a release film) or remain relatively exotic (e.g. tool surface patterning) and unlikely to be quickly implemented in an existing production environment.

\subsection{Objectives}


In this work, we clarify the fundamental causes of surface porosity during VBO prepreg processing and identify avenues for mitigating surface defects that avoid adding significant manufacturing time or part weight. We describe a systematic experimental study consisting of material characterization, manufacturing trials and surface porosity quantification. The resulting data clarify the dominant material-process-property relationships, and identify science-based approaches for effectively and consistently reducing surface defects.

\section{Experimental Procedures}

\subsection{Test Matrix}

Table 1 outlines the factors considered, the ranges investigated, and the prepreg material used. These factors include both material properties and manufacturing parameters.

The fiber bed architecture we considered included typical woven fabric and unidirectional product forms to determine the influence of prepreg surface topology on surface void formation during VBO processing. Prepregs were conditioned in humid ambients to determine the effect of absorbed moisture within the resin on surface porosity. Prepreg plies were conditioned for 30 hours on a tray within a sealed bell jar partially filled with water below the tray. The water gave rise to a constant relative humidity level of $99 \%$, confirmed by a digital humidity sensor. A four-hour vacuum hold at $20^{\circ} \mathrm{C}$ was performed before cure to allow sufficient time for all air trapped during layup to be evacuated, thus ensuring that any surface defects present were a result of moisture, not trapped air. A wide range of out times was selected to determine the effects of increased resin viscosity and decreased prepreg tack on the formation and evolution of surface pores. Prepreg plies were aged at ambient conditions while stored within sealed plastic bags, as well as after being placed on the tool, to determine whether the state of the prepreg at the moment of layup was significant. Finally, the influence of the freezer 
storage time was investigated by fabricating laminates with material at the beginning and end of the stated life (12 months). In all cases, frozen prepreg was stored in sealed bags provided by the material manufacturer at a temperature below $-23^{\circ} \mathrm{C}\left(-10^{\circ} \mathrm{F}\right)$.

Layup was performed using both liquid and film release methods. The room temperature (RT) vacuum hold time was varied by subjecting parts to vacuum for zero to sixteen hours prior to high temperature cure to evaluate the influence of air evacuation on surface pore formation. In addition to RT vacuum holds, two additional methods of extracting air from the tool-part interface were performed: a ten-minute intermediate debulk on the first ply and spiking of the first ply. Debulking involved assembling a temporary vacuum bag around the first ply and applying vacuum consolidation before laying up the remaining plies. Spiking entailed rolling a spiked roller tool to create a regular pattern of transverse perforations within the prepreg. Finally, we investigated the influence of vacuum quality by fabricating laminates using full vacuum (denoted as $99 \mathrm{kPa}$, and corresponding to an absolute bag pressure of $\sim 2.3 \mathrm{kPa}$ ) and $80 \%$ vacuum (denoted as $80 \mathrm{kPa}$, and corresponding to a bag pressure of $\sim 21.3 \mathrm{kPa}$ ).

Within a given set of results, we varied only one parameter. Any baseline case that is presented was a baseline unique to the individual parameter being discussed. This ensured that any parameter not considered in each individual case did not play a role in the results. For example, when we investigated the effects of prepreg out time, we used the same room temperature vacuum hold time, freezer time, moisture content, fiber architecture, and release method for all laminates. The only parameter we varied was out time, and we fabricated the baseline laminates using unaged prepreg (no out time). 


\subsection{Materials}

The experiments described below were performed on two representative carbon fiber/epoxy VBO prepregs designed for primary structure applications. They consisted of a toughened epoxy (Cytec Industries, Inc. 5320-1) and two fiber beds: an eight-harness satin woven fabric and a unidirectional tape (UD). The fiber bed areal weight, resin content and cured ply thickness of each material are provided in Table 2. The 5320-1 resin is designed for cure at $93^{\circ} \mathrm{C}$ or $121^{\circ} \mathrm{C}$, for three or twelve hours, respectively. A RT vacuum hold ranging from four to sixteen hours is also recommended, depending on the part size. The specified out life of the resin is 30 days, and the freezer life is one year from date of manufacture.

All laminates were fabricated on the same aluminum tool. Prior to use, the tool surface was prepared through orbital sanding with 80 -grit sandpaper. The tool surface roughness was $0.76 \mu \mathrm{m}$, measured using a surface profilometer (Zygo NexView 8000).

\subsection{Laminate Manufacturing}

Laminates were cured by VBO processing in an air-circulating oven (Thermal Product Solutions Blue M). The cure cycle was consistent with manufacturer recommendations, and consisted of a RT vacuum hold of varying length, $1.5^{\circ} \mathrm{C} / \mathrm{min}$ ramp rates, and a three hour dwell at $121^{\circ} \mathrm{C}$. The vacuum bag assembly, shown in Figure 1, consisted of a tool treated with a release agent (liquid or film), the laminate, a bag-side non-perforated release film, edge breathing dams made of sealant tape wrapped in fiberglass boat cloth, a layer of breather and the vacuum bag. For liquid release, a commercial agent (Frekote 770-NC) was applied to the tool three times in succession, allowing each coat to dry five minutes before applying the next layer. After the third coat, the tool plate was dried for thirty minutes before laying up the laminate. After every third laminate produced with the tool plate, the tool was wiped with acetone and release agent was 
reapplied. For film release, a single ply of non-perforated fluorinated ethylene propylene (FEP) was placed at the tool-part interface.

The size and stacking sequence of each laminate was $127 \mathrm{~mm} \times 127 \mathrm{~mm}$ and 4 plies stacked $[0 / 90]_{\mathrm{S}}$ respectively. In total, 106 laminates were produced for this study; the number of repeat laminates produced for each testing condition is outlined in Table 1 . The surface porosity values reported below are averages.

\subsection{Surface Porosity Measurement}

Surface porosity was measured using a low-magnification, handheld microscope (DinoLite Premier2 Digital Microscope). Sixteen images of each laminate surface were recorded at $20 \times$ magnification and analyzed using software (ImageJ). Each image spanned an area of 180 $\mathrm{mm}^{2}$. The procedure used to analyze the images is shown in Figure 2. Raw images were imported into the program and all areas of surface porosity were manually selected. Using the software, the micrographs were converted to binary images with areas of surface porosity black, and the remaining area white. A percent value for surface porosity was determined by dividing the number of black pixels by the total number of pixels in the image. The sixteen values calculated for each laminate were averaged to obtain an average surface porosity value for each part. Finally, the average surface porosity values of a set of repeat laminates were averaged to determine the average surface porosity for a given experimental condition.

\subsection{Visual Analysis}

The surface topography of uncured prepreg was measured using an optical surface profilometer (Zygo NexView 8000). Three-dimensional topography maps were obtained, with $x$ $y$ dimensions of $9.1 \mathrm{~mm} \times 7.8 \mathrm{~mm}$, and a surface roughness $\left(S_{a}\right)$ value for each prepreg was 
measured. Micrographs of prepreg surfaces and cross sections were acquired using a scanning electron microscope (JEOL 6610) in backscatter mode, at an accelerating voltage of $20 \mathrm{kV}$.

To better understanding the mechanism of air evacuation and air entrapment, four $75 \mathrm{~mm}$ $\times 75 \mathrm{~mm}$ prepreg plies were laid up with a $[0 / 90]_{\text {s }}$ stacking sequence on a glass plate to simulate a tool-prepreg interface that could be observed directly. This procedure has been used in previous studies to evaluate air entrapment, distribution, and migration, both for internal porosity [9] and surface porosity [12]. Time-lapse images were recorded with a portable microscope (Dino-Lite Premier2 Digital Microscope, 20×) while the assembly was held under vacuum. These images clearly showed the location, size and evolution of gaps (or bubbles) between the resin-rich surface of the tool-side prepreg ply and the surface of the glass tool under vacuum compaction. These gaps, and their gradual collapse, are likely correlated to the presence and evacuation of air entrapped at the tool-part interface.

\section{Results and Discussion}

\subsection{Source of Surface Porosity}

We investigated the effect of prepreg fiber architecture to explore the possibility that surface porosity might stem from air trapped at the tool-prepreg interface during layup. Laminates fabricated from woven prepreg exhibited extensive surface porosity, including both circular and square-like pores ranging in size from 0.004 to $1.220 \mathrm{~mm}^{2}$ (Figure 3a). In contrast, no surface porosity was observed in any laminates produced with UD prepreg, as shown in Figure $3 b$. The distinction is attributed to the different surface topography of the prepregs associated with the two fiber architectures.

Figure 4 shows the quantitative topography of the woven and UD prepregs prior to laminating. The linear roughness profiles of each prepreg (Figures $4 c$ and d) highlight the 
differences between the two types of prepregs. Woven prepregs exhibit a periodic pattern of hillocks and valleys corresponding to the overlaps and underlaps of tows in the weave, the height differences ranging from 100-200 $\mu \mathrm{m}$. When placed on the flat tool plate, the overlaps of the prepreg will touch the tool, while the underlaps will remain slightly raised, creating regions in which air can be trapped. Conversely, the UD prepreg presents a much smoother surface, with an areal roughness of only $12.7 \mu \mathrm{m}$ (compared to $58.6 \mu \mathrm{m}$ for the woven prepreg), leading to less air initially trapped at the tool-prepreg interface.

To directly observe air entrapment, the mechanism of air evacuation, and the conformation of the prepreg to the tool surface, we laid up a small laminate on a glass plate, thereby simulating a tool-prepreg interface while affording transparency. Time-lapse images of the system under vacuum are shown in Figure 5. Figure 5a shows the interface immediately after applying vacuum. The gaps (or bubbles) outlined in black are largely located within the gaps formed by underlap regions of the woven prepreg. Under vacuum compaction, these gaps become air bubbles trapped at the tool-prepreg interface. Figures 5b-d show that the sizes and positions of the bubbles changed over time under vacuum. During the room temperature vacuum hold, the bubbles decreased in size and remained relatively stationary. Occasionally, bubbles moved slightly, always toward sites of tow underlap, indicating that the trapped air was evacuated out-of-plane at tow underlaps. As the bubbles decreased in size, the resin-rich surface of the prepreg gradually contacted the surface of the tool. Any bubbles remaining after resin gelation would have formed surface porosity.

SEM micrographs reveal open slits in the woven prepreg that provide an evacuation route for trapped air (Figures 6a and b). A cross section shows the dry fiber tows present in the woven OoA prepregs (Figure 6c), known as engineered vacuum channels (EVaCs). In the manufacture 
of OoA prepregs, resin films are applied to dry fabric to partially impregnate fibers from top and bottom surfaces. Consequently, dry fiber tows (as shown in Figure 6c) reside directly beneath surface layers of resin, and these dry fibers are specifically designed to provide pathways for air evacuation from the laminate while under vacuum. Thus, the open slits shown in Figures 6a and b provide external access to the dry fiber tow cores (EVaCs), facilitating the evacuation of air trapped at the tool-prepreg interface. In particular, we observed bubbles moving toward these pinhole openings during the vacuum hold, indicating that the vacuum within the dry EVaCs can contribute to the evacuation of air from the tool-part interface. Note, however, that air flow was not directly measured in this study.

We also investigated the effects of absorbed moisture on surface porosity, which reportedly can lead to porosity in cured laminates produced from OoA prepregs $[13,14]$. Inspection of tool-side surfaces of laminates conditioned at different humidity levels revealed defects distinctly different from the shape and distribution of typical surface porosity (Figure 7). The surface defects took the form of pits uniformly distributed across the entire laminate, unlike surface pores in "dry" laminates, which were typically restricted to resin-rich areas between tows. Moisture in the prepreg did not eliminate surface porosity due to trapped air, as this type of surface porosity was observed on conditioned laminates (Figure 7). However, a magnified view highlights the difference between the new, pit-like surface defects that were observed and the bubble-like surface porosity known to result from trapped air (Figure 7b). A more comprehensive study of this parameter is required to draw definitive conclusions about moisturerelated surface porosity. However, these results indicate that the most common type of surface porosity - large surface pores in resin-rich tow overlap locations - arises from entrapped air.

\subsection{Material and Process Effects}


The effects of select material and processing parameters were investigated to determine the effect of each factor on air entrapment and/or removal. The parameters selected included RT vacuum hold time, prepreg out time and freezer storage time, prepreg permeability, and vacuum level. These parameters are inevitable consequences of the need for freezer storage and common layup processes used in laminate fabrication, all of which can increase the risk of porosity in general. Mitigation strategies were identified through systematic investigation of the parametric effects.

\subsubsection{Room Temperature Vacuum Hold Effects}

We observed an inverse correlation between surface porosity and RT vacuum hold time. In particular, as vacuum hold time increased, the amount of surface porosity exhibited by the cured laminate decreased (Figure 8a). These results confirmed the original hypothesis that was based on visual observation - that surface porosity is linked to entrapped air. Because air evacuation is a time-dependent process, decreasing the room temperature vacuum hold decreased the opportunity for trapped air to escape.

\subsubsection{Out Time Effects}

The effect of prepreg out time on surface porosity was pronounced, as shown in Figure 8b. The amount of surface porosity decreased by $83 \%$ after just four days of out time and by $99 \%$ after 14 days of out time. The decrease in surface porosity with increased out time was attributed to the concurrent decrease in prepreg tack and ply compliance, and to the increase in ambient temperature resin viscosity associated with RT aging of prepregs [15]. When prepreg tack decreases, the adhesive bond between the prepreg and other objects, particularly the tool plate, also decreases. This has been confirmed in previous studies through energy of separation tests, performed by pressing together two plies of prepreg, and subsequently measuring the 
energy required to separate them $[15,16]$. Prepreg tack affects adhesion strength [16] and varies with fluidity of the resin [17]. A tackier resin forms a stronger adhesive bond to an opposing surface or substrate. The increase in resin viscosity and decrease in ply compliance with out-time have also been previously documented, and decrease the capacity (1) of the resin to flow, and (2) of the ply to deform under load [17,18]. These factors affect surface porosity as described below.

When a prepreg with low out-time (high tack, low resin viscosity and high compliance) is compacted under vacuum at ambient temperature, the resin-rich surface can adhere and conform to the tool surface. In particular, the resin can flow locally within the gaps formed by the tow overlaps, potentially closing off in-plane air evacuation pathways within the interface and out-ofplane pathways between the interface and the EVaCs located within the dry fiber tows. As a result, air bubbles can remain entrapped at the tool-part interface during cure, leading to surface porosity. Conversely, for a prepreg with higher out-time (low tack, high resin viscosity and low compliance), vacuum compaction does not lead to the same degree of conformation to the tool surface. The lower flow capacity of the resin-rich regions, coupled with the stiffer saturated fiber bed regions, allow the prepreg to retain an uneven surface topology. As a result, comparatively more air evacuation pathways remain open, increasing the interface permeability relative to the low out-time case [19] and enhancing the evacuation of entrapped air. During subsequent high temperature ramp, the resin viscosity decreases by orders of magnitude, allowing the resin-rich ply surface to conform to the tool and resulting in a uniform, void-free surface. Thus, increasing out time leads to a decrease in surface porosity. While contrary to intuition, these trends are nevertheless consistent with previous reports that increased out time led to a decrease in internal (macro) void content in laminates, a phenomenon attributed to increased in-plane permeability $[15,20,21]$. 
To specifically separate the effects of reduced tack from the other consequences of out time (i.e. resin viscosity and ply compliance), four laminates were cured at the same time on the same tool plate from prepreg with equal out time. The only difference between the laminates was the aging process prior to cure. Two of the laminates were fabricated from prepreg aged for five days in a sealed bag prior to lay-up on the tool plate. The other two laminates were fabricated using prepreg laid up on the tool plate at zero days out time and allowed to age on the tool plate for five days prior to oven cure. Figure $8 \mathrm{~d}$ shows that the two laminates laid up on the tool plate at zero days and allowed to age on the tool exhibited greater surface porosity than the laminates aged prior to lay-up on the tool. The observations indicate that the observed decrease in surface porosity resulted primarily from the reduced tack associated with out time.

\subsubsection{Freezer Time Effects}

The effect of freezer storage time on surface porosity was investigated by aging prepreg sealed in bags in a freezer (less than $-23^{\circ} \mathrm{C}$ ) for six and twelve months before being laid up on a tool plate to cure. The results, shown in Figure 8c, demonstrated that surface porosity generally decreased with freezer storage time - freezer storage for six months resulted in a $93 \%$ decrease in surface porosity. The finding is consistent with the observed decrease in surface porosity resulting from out time, and is again attributed to reduced tack. Previous studies have shown that even at freezer temperatures, epoxy resins undergo cross-linking and slowly cure, thus reducing tack [17]. At 12 months and zero vacuum hold time, the trend reverses, and surface porosity appears in laminates produced at this testing condition. However, the amount of surface porosity is still less than observed when using fresh prepreg, and 12 months marks the end of the specified shelf life of the prepreg, the maximum allowable storage life at $-18^{\circ} \mathrm{C}$ or lower.

\subsubsection{Processing Effects}


The effects of several processing variations on surface porosity were also considered and are summarized in Figure 9. The layup of large or complex parts often includes intermittent debulking periods. These short, periodic applications of vacuum help individual plies conform to the tool shape and can also evacuate entrapped air. In the present study, intermittent debulks of the first (tool-side) ply produced no change in surface porosity. A second process variation involved spiking the prepreg plies with a spiked roller tool before laying up the laminate to increase through-thickness permeability. As a result, surface porosity was reduced by $40 \%$, and the variance decreased five-fold. Although a more comprehensive study of the effect of out-ofplane permeability on surface porosity is required to draw definitive conclusions about the effects of this parameter, the procedure of prepreg spiking does not appear to completely eliminate surface porosity. Spiking can also lead to fiber breakage and reduce mechanical performance.

Finally, we investigated the effects of reduced vacuum on surface porosity. Vacuum level is a key parameter in the manufacture of composite parts, and in production settings, bagged parts commonly undergo changes in vacuum level resulting from bag size, bag leaks, and the fact that different bags are often connected and disconnected from the same pump. Figure 9 compares the results from three laminates cured at $80 \mathrm{kPa}$ (reduced vacuum) to the results of control samples cured at $99 \mathrm{kPa}$ (full vacuum). A 20\% decrease in vacuum level resulted in an almost $200 \%$ increase in surface porosity. This result aligns with findings of previous studies and demonstrates the importance of maintaining high vacuum when curing composite parts, particularly out of autoclave [22-26]. 


\subsubsection{Statistical Significance}

The $t$-test is a common method used to determine if two sets of data are significantly different from each other. However, there is disagreement in the literature over whether or not ttests can be performed on sample population sizes less than five [27]. Due to time and material restrictions, our sample sets were typically restricted to smaller population sizes $(\mathrm{N}=3)$. Nevertheless, we present the results of unpaired, two-sample $t$-tests between the control conditions and each respective deviating condition. For example, a $t$-test was performed between the mean of the control (zero out time) and the mean of the laminates produced at six days of out time to determine the significance of the decrease in surface porosity observed with six days of out-time.

The difference in means of two sample sets is considered statistically significant if the $p$ value determined from the $t$-test is less than the chosen threshold value (usually 0.05) [27]. All out-time levels were deemed statistically significant $(p<0.05)$ except for the four-day level, where the $p$-value was 0.053 . The results presented for aging method (allowing prepreg to age off the tool vs. on the tool) were less significant, with a $p$-value of 0.076 . However, these particular data sets had an extremely small sample size of $\mathrm{N}=2$. The increase in surface porosity with decreased vacuum level in the bag was statistically significant with a $p$-value of 0.018 , indicating that vacuum quality was a critical factor for defect suppression. Similarly, the length of RT vacuum hold had a statistically significant affect on surface porosity, as a $t$-test between laminates fabricated from fresh prepreg with no RT vacuum hold and those that underwent a 16hour hold resulted in a $p$-value of 0.042 . Finally, a $p$-value of 0.038 was obtained when comparing laminates fabricated from fresh prepreg to laminates made from prepreg with six months of freezer time, marking the result as statistically significant. The remaining results 
associated with freezer time were statistically less significant due to the coupled effect of the room temperature vacuum hold, which prevented, in some cases, the formation of surface porosity in both control and aged laminates.

\subsection{Mitigation Strategies}

In this study, we investigated surface porosity as a function of material and process factors. For the chosen materials and part size, surface porosity was observed only for woven fabric prepreg laminates manufactured on tools coated with liquid release agents. These results, in conjunction with visual observations of laminates compacted on a glass tool plate, indicated that surface porosity was typically caused by air entrapped at the tool-part interface, within gaps formed by the uneven surface of woven fabric prepregs.

Prepreg out time was identified as a major factor affecting surface porosity due to its influence on air evacuation. High out time leads to reduced tack and ply compliance and increased resin viscosity. The consequent decrease in conformation between the prepreg and tool surface increases the ability to evacuate air entrapped at the interface relative to the baseline case with no out time. For this study, surface porosity was largely eliminated for out times greater than five days. The effects of freezer time were shown to be statistically less significant.

The quality of the vacuum drawn in the bag was also identified as critical: higher bag pressures reduce the driving force for air evacuation while directly causing more air to be present in the bag, leading to higher surface porosity. The results also confirmed that, as intuited, longer room temperature vacuum hold times reduced surface porosity levels by extending the time available to evacuate air prior to cure.

Two modifications designed to improve air evacuation were investigated: debulking and spiking the first ply. Both protocols improved surface porosity levels but had low statistical 
significance. Finally, the effect of humidity conditioning was studied for a single sample, which exhibited surface porosity with different characteristics (pore size, shape and distribution) than that observed in all other samples, further supporting the conclusion that typical surface porosity is mostly associated with air entrapment at the tool-part interface.

Of the various measures we investigated to mitigate the effect of entrapped air on surface porosity, two were shown to be effective: (1) maintaining a high vacuum level in the bag, and (2) using prepreg with reduced tack for the first (tool-side) ply. Both measures reduce air entrapment and/or foster air evacuation. The first method increases the driving force for air evacuation, while the second reduces the barrier to evacuation. Using prepreg with reduced tack raises two concerns. First, reduced tack will increase the risk of plies slipping out of position as they are laid up. Despite this concern, using prepregs with four days of out time significantly reduced surface porosity, and this out time falls well within both the thirty-day out life and the twentyday tack life of the material. Second, the use of reduced tack prepreg increases the risk that unacceptable amounts of internal porosity will result in the laminate. As prepreg out time and/or freezer time increases, resin viscosity also increases [28], impeding tow impregnation and potentially leading to micro-voids in cured laminates. However, previous research has shown that the void content does not begin to noticeably increase until 14 days of out time, whereas surface porosity decreases to nearly zero by the 14-day mark [17].

In the commercial production of composite parts, surface porosity is often encountered intermittently - some laminates exhibit extensive surface porosity, while others fabricated by a similar process exhibit negligible surface porosity. The results described here provide insight into this phenomenon. Nearly all of the data exhibit large coefficients of variation (roughly 20$100 \%$ ), which reflects the variability from panel to panel and the inconsistent and localized 
nature of air entrapment. In addition, a multitude of other factors can contribute to intermittent surface defects in a production environment, most notably, variability in vacuum quality due to multiple parts connected to the same vacuum pump, and minor but not insignificant differences in prepreg out times, particularly between zero and five days. Consistent control of surface porosity in laminates fabricated with OoA prepregs requires both an understanding of the fundamental causes and a practice of tracking requisite processing and material parameters.

\section{Conclusions}

In this study, we demonstrated the roles of several material and processing parameters in surface porosity formation and mitigation. Air trapped during the layup process at the toolprepreg interface was identified as the source of large surface pores located in resin-rich regions of the cured composite tool-side surface. Moisture present in the prepreg prior to cure also played a role in surface defects, but these defects were distinct from the surface porosity typically caused by entrapped air. Moisture effects were not the primary focus of this particular study, and further investigation is required to draw conclusions about the formation and mitigation of surface defects that arise from absorbed moisture in the prepreg. For surface porosity that arises from entrapped air, results confirm that vacuum level in the bag and prepreg freezer and out time are the primary drivers of effective mitigation.

As composite applications expand into new sectors of industry, surface appearance will continue to play a major role in consumer acceptance (e.g automotive, recreational equipment, wind energy, gas storage, etc.). Non-autoclave cure will only be successful if the defects normally suppressed by autoclave pressure continue to be absent, and both performance and appearance are comparable to parts processed in the autoclave. Because of the absence of high pressure to suppress the multiple sources of defects, imperfections, and variability, OoA prepregs 
are particularly susceptible to air entrapment and gas-induced voids. However, all sources of porosity can be controlled/eliminated by developing prepregs with increased air evacuation (effective permeability). A key finding of this study is that prepreg out time increases in-plane permeability of the tool-prepreg interface, and that using prepreg with just a few days of out time, at least for the tool-side surface ply, can effectively eliminate surface porosity. These defect-reduction strategies can improve manufacturing efficiency and sustainability by reducing the number of steps required to produce a part, a widespread objective within the composites industry.

\section{Acknowledgements}

The authors are grateful for financial support from the National Science Foundation, through the G8 Research Councils Multilateral Initiative on Materials Efficiency and the "Sustainable Manufacturing Through Out-of-Autoclave Prepregs" project (NSF CMMI-1229011). The authors also thank Doug Decker at Northrop Grumman Corporation for his useful advice, as well as Cytec Industries Inc. and Airtech International for prepreg and consumable material donations. Zygo Corporation provided access to their equipment, for which the authors are very grateful. Discussions with Dr. Gaurav Nilakantan are greatly appreciated. The authors also thank undergraduate researcher Christina Cabble for her efforts and assistance throughout the project. Finally, the project's partner academic institutions (the University of Bristol, the Technical University Munich, and McGill University) as well as its industrial advisory board are acknowledged for their input. 


\section{References}

[1] Centea T, Grunenfelder LK, Nutt SR. A review of out-of-autoclave prepregs Material properties, process phenomena, and manufacturing considerations. Compos Part A Appl Sci Manuf 2015;70:132-54.

[2] Herring ML, Fox BL. The effect of a rapid curing process on the surface finish of a carbon fibre epoxy composite. Composites Part B 2011;42(5):1035-43.

[3] Darrow DC, Poropatic PA, Brayden TH. Elimination of mold surface porosity on composite parts. J Adv Mater 1995;27(1):41-6.

[4] Ridgard, C. Out of autoclave composite technology for aerospace, defense and space structures. Proc SAMPE 2009 conf. Baltimore, MD: Society for the Advancement of Materials and Process Engineering; 2009.

[5] Herring ML, Mardel JI, Fox BL. The effect of material selection and manufacturing process on the surface finish of carbon fibre composites. J Mater Process Technol 2010;210(6):926-40.

[6] Hamill L, Centea T, Nilakantan G, Nutt SR. Surface porosity in out-of-autoclave prepreg processing: causes and reduction strategies. Proc SAMPE 2014 conf. Seattle, WA: Society for the Advancement of Materials and Process Engineering; 2014.

[7] Grigoriev MM, Han J, Woo R, Cheng D. Effect of atmospheric pressure plasma treatment on surface porosity of OOA composites. Proc SAMPE 2013 conf. Redondo Beach, CA: Society for the Advancement of Materials and Process Engineering; 2013.

[8] Grigoriev MM, Hogikyan A, Cheng D. Porosity-free molded surfaces for out-ofautoclave composites. Proc SAMPE 2014 conf. Seattle, WA: Society for the Advancement of Materials and Process Engineering; 2014.

[9] Cender TA, Simacek P, Advani SG. Resin film impregnation in fabric prepregs with dual length scale permeability. Compos Part A Appl Sci Manuf 2013;53:118-28.

[10] Gangloff JJ, Daniel C, Advani SG. A model of two-phase resin and void flow during composites processing. Int J Multiph Flow 2014;65:51-60.

[11] Gangloff JJ, Cender TA, Eskizeybek V, Simacek P, Advani SG. In situ flow visualization of void migration during out-of-autoclave thermoset prepreg processing. Proc. $12^{\text {th }}$ Int. Conf. Flow Process. Compos. Mater., Twente, Netherlands: 2014, p.1-2.

[12] Bloom LD, Napper MA, Ward C, Potter K. On the evolution of the distribution of entrapped air at the tool/first ply interface during lay-up and debulk. Adv Manuf Polym Compos Sci 2014;1:36-43.

[13] Grunenfelder LK, Nutt SR. Void formation in composite prepregs - Effect of dissolved moisture. Compos Sci Technol 2010;70(16):2304-9.

[14] Kardos JL, Dudukovic MP, Dave R. Void growth and resin transport during processing of thermosetting-matrix composites. Adv Polym Sci 1986;80:102-23

[15] Grunenfelder LK, Nutt SR. Out time effects on VBO prepreg and laminate properties. Proc SAMPE 2011 conf. Long Beach, CA: Society for the Advancement of Materials and Process Engineering; 2011.

[16] Ahn KJ, Peterson L, Seferis JC, Nowacki D, Zachmann HG. Prepreg Aging in Relation to Tack. J Appl Polym Sci 1992;45(3):399-406.

[17] Grunenfelder LK, Centea T, Hubert P, Nutt SR. Effect of room-temperature out-time on tow impregnation in an out-of-autoclave prepreg. Compos Part A Appl Sci Manuf 2013;45:119-26. 
[18] Centea T, Kratz J, Hubert P. The effect of out-time and cure cycle on the consolidation of out-of-autoclave prepregs. Proc. $11^{\text {th }}$ Int. Conf. Flow Process. Compos. Mater., Auckland, New Zealand: 2012.

[19] Putnam JW, Seferis JC. Prepreg gas permeation as a function of fiber orientation and aging time. J Adv Mater 1995;26(3):36-41.

[20] Centea T, Hubert P. Measuring the impregnation of an out-of-autoclave prepreg by micro-CT. Compos Sci Technol 2011;71(5):593-99.

[21] Putnam JW, Seferis JC. Prepreg Gas Permeation as a Function of Fiber Orientation and Aging Time. J Adv Mater 1995;26(3):35-41.

[22] Grunenfelder LK, Nutt SR. Moisture and Pressure Effects on Void Formation in Prepreg Processed Composites. Proc ASC 2010 conf. Dayton, OH: American Society for Composites; 2010.

[23] Tang J-M, Lee WI, Springer GS. Effects of cure pressure on resin flow, voids, and mechanical properties. J Compos Mater 1987;21(5):421-40.

[24] Liu L, Zhang B-M, Wang D-F, Wu Z-J. Effects of cure cycles on void content and mechanical properties of composite laminates. Compos Struct 2006;73(3):303-09.

[25] Centea T, Hubert P. Out-of-autoclave prepreg consolidation under deficient pressure conditions. J Compos Mater 2013;48(16):2033-45.

[26] Boey FYC, Lye SW. Void reduction in autoclave processing of thermoset composites - Part 1: High pressure effects on void reduction. Composites 1992;23(4):261-65.

[27] de Winter DCF. Using the Student's t-test with extremely small sample sizes. Practical Assessment, Research \& Evaluation 2013;18(10):1-12.

[28] Gu Y, Li M, Zhang Z, Li Y. Effects of resin storage aging on rheological property and consolidation of composite laminates. Polym Compos 2009;30(8):1081-90. 


\section{Figure Captions}

Figure 1. Vacuum bag assembly for laminate manufacture.

Figure 2. Sequence of actions used to quantify surface porosity. (a) Raw image taken at 20x magnification is uploaded into image analysis software; (b) areas of surface porosity are manually outlined; (c) image is converted into binary image where black pixels represent areas of surface porosity. The black pixels in this image represent $1.43 \%$ of the total pixels in the image, translating to $1.43 \%$ of this area of laminate surface being surface porosity.

Figure 3. Typical shape and distribution of surface porosity observed on laminates made from (a) woven and (b) unidirectional prepreg. Note that no surface porosity is present on laminates made from unidirectional prepreg, whereas surface porosity is widely present on laminates made from woven prepreg.

Figure 4. Surface topography of the (a) woven and (b) unidirectional prepreg used in this study. Roughness lines given in (c) and (d) correspond to the white lines in (a) and (b) respectively. Figure 5. Time-lapse images of a glass plate-prepreg interface under vacuum at room temperature. Images were taken (a) immediately after application of vacuum as well as (b) 10 minutes, (c) 30 minutes, and (d) 1 hour in to the vacuum hold. Select air bubbles are circled to highlight the progression of shape, size and location of trapped air.

Figure 6. SEM micrographs of the woven prepreg surface. An open slit in the resin is shown at (a) 22x and (b) $75 x$. (c) SEM micrograph taken at 25x of a cross section of two stacked woven prepreg plies. Dry fiber bundles are observed directly below the surface resin.

Figure 7. (a) Tool-side surface of a laminate made from woven prepreg plies conditioned in a humidity chamber prior to layup and cure alongside a (b) magnified view, which highlights the difference between porosity due to trapped air and surface defects resulting from moisture in the prepreg.

Figure 8. The effect of (a) room temperature vacuum hold time, (b) out time at room temperature, and (c) freezer time on surface porosity. (d) The effect of room temperature aging method on surface porosity. "Control" laminates were made from prepreg with no out time; "On Tool" laminates were made from prepreg that was laid up on the tool at zero days of out time and allowed to age for five days prior to cure; "Off Tool" laminates were made from prepreg that was aged for five days prior to being laid up and were cured immediately upon layup.

Figure 9. The effect of additional processing parameters on surface porosity was considered. These included performing an intermittent debulk on the first ply down, spiking the first ply down, and reducing the vacuum level. 
Tables

Table 1. Parameters, ranges, prepreg choice and number of repeat tests included in parametric study.

\begin{tabular}{|c|c|c|c|}
\hline Parameter & Range & Prepreg & $\begin{array}{l}\text { Number of } \\
\text { Repeat Tests }\end{array}$ \\
\hline \multicolumn{4}{|c|}{ Material } \\
\hline Prepreg Fiber Bed Architecture & Unidrectional; Woven & UD; Woven & 3 \\
\hline Prepreg Humidity Conditioning & $99 \% \mathrm{RH}$ & Woven & 3 \\
\hline Prepreg Out Time & 0-49 Days & Woven & 3 \\
\hline Out Time Aging Method & On tool; Off tool & Woven & 2 \\
\hline Prepreg Freezer Time & 0-12 Months & Woven & 3 \\
\hline \multicolumn{4}{|c|}{ Processing } \\
\hline Release Method & Liquid Agent; Film & UD; Woven & 3 \\
\hline Room Temperature Vacuum Hold & $0-16$ Hours & Woven & 3 \\
\hline Debulk $1^{\text {st }}$ Ply Down & 0-10 Minutes & Woven & 3 \\
\hline Spike $1^{\text {st }}$ Ply Down & None; Spiking & Woven & 3 \\
\hline Reduced Vacuum Level & $80-99 \mathrm{kPa}$ & Woven & 3 \\
\hline
\end{tabular}


Table 2. Prepreg and tool plate material properties.

\begin{tabular}{|c|c|c|c|c|c|c|}
\hline \multicolumn{7}{|c|}{ Prepreg } \\
\hline $\begin{array}{l}\text { Short } \\
\text { Name }\end{array}$ & $\begin{array}{c}\text { Fiber } \\
\text { Architecture }\end{array}$ & Fiber & $\begin{array}{c}\text { Fiber Bed } \\
\text { Areal } \\
\text { Weight } \\
\left(\mathbf{g} / \mathbf{m}^{2}\right)\end{array}$ & Resin & $\begin{array}{c}\text { Resin } \\
\text { Content } \\
(w t \%)\end{array}$ & $\begin{array}{c}\text { Cured Ply } \\
\text { Thickness } \\
\text { (mm) }\end{array}$ \\
\hline Woven & $\begin{array}{l}8 \text { Harness } \\
\text { Satin }\end{array}$ & Carbon & 370 & Epoxy & 36 & 0.39 \\
\hline UD & Unidirectional & Carbon & 145 & Epoxy & 33 & 0.14 \\
\hline \multicolumn{7}{|l|}{ Tool } \\
\hline $\begin{array}{l}\text { Short } \\
\text { Name }\end{array}$ & Material & Roughness & & & & \\
\hline O80 & Aluminum & $0.76 \mu \mathrm{m}$ & & & & \\
\hline
\end{tabular}




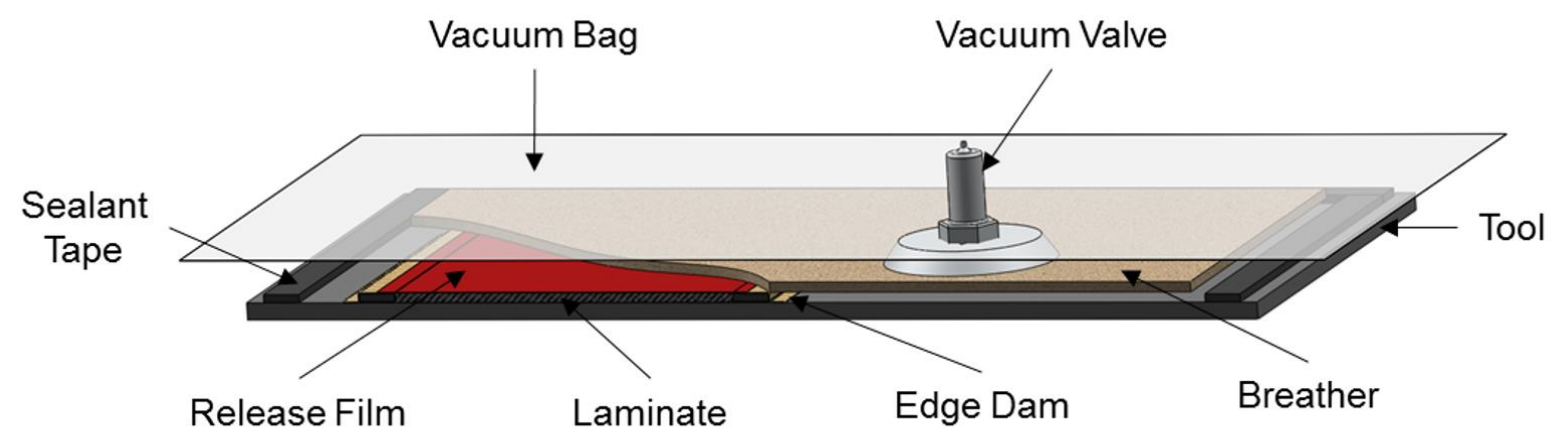




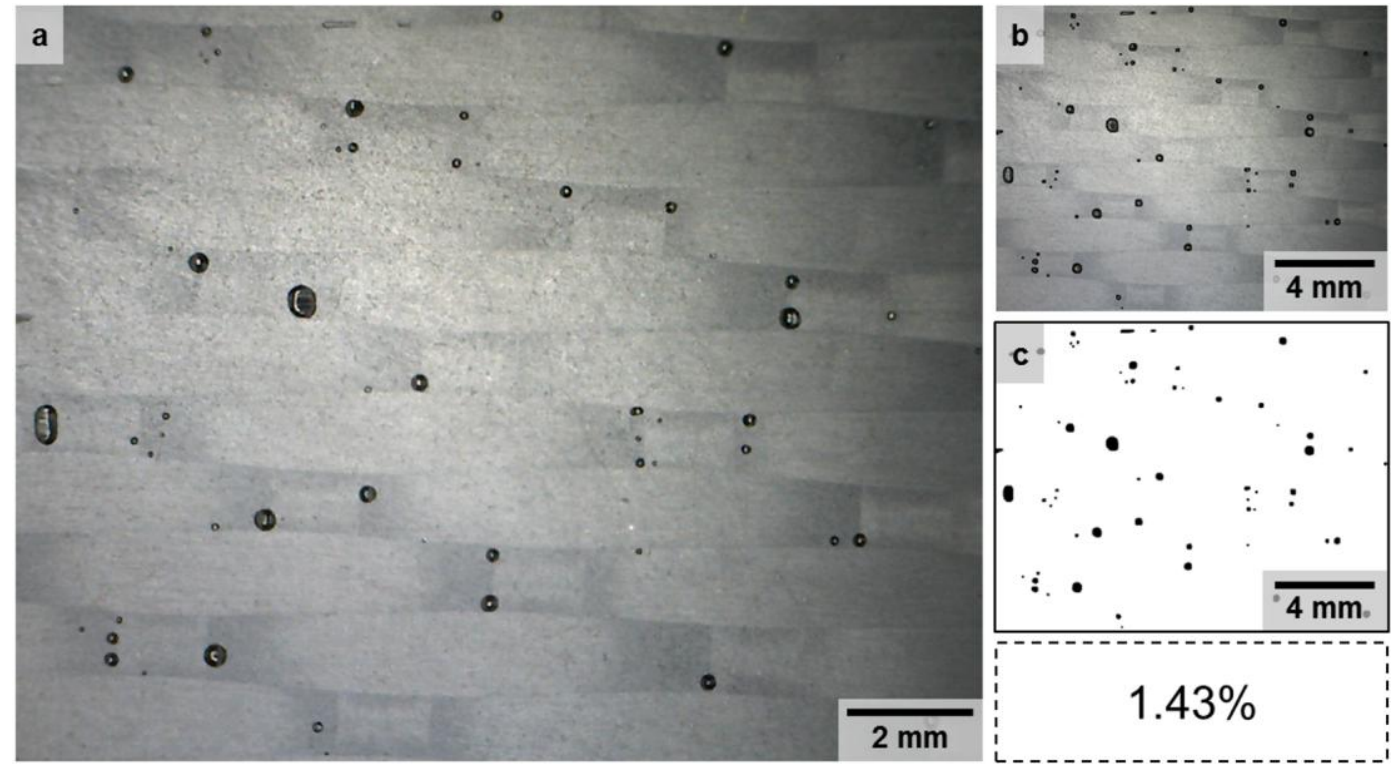




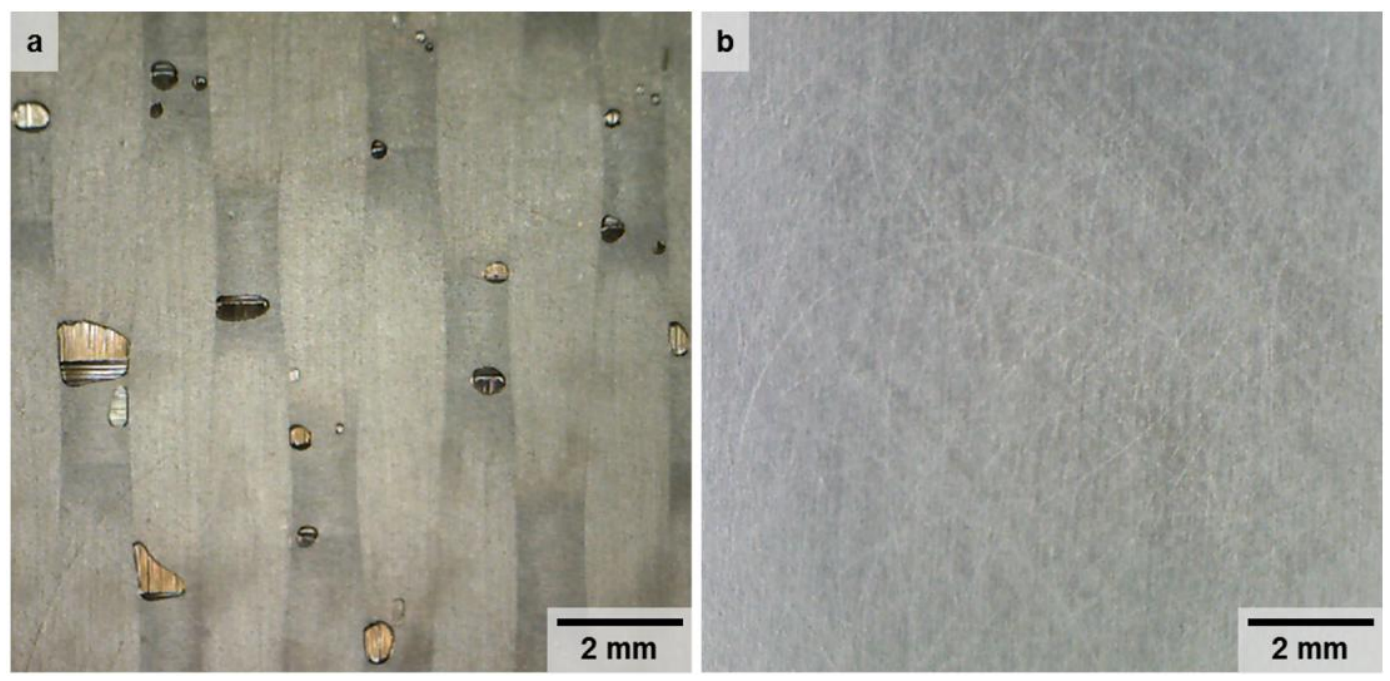



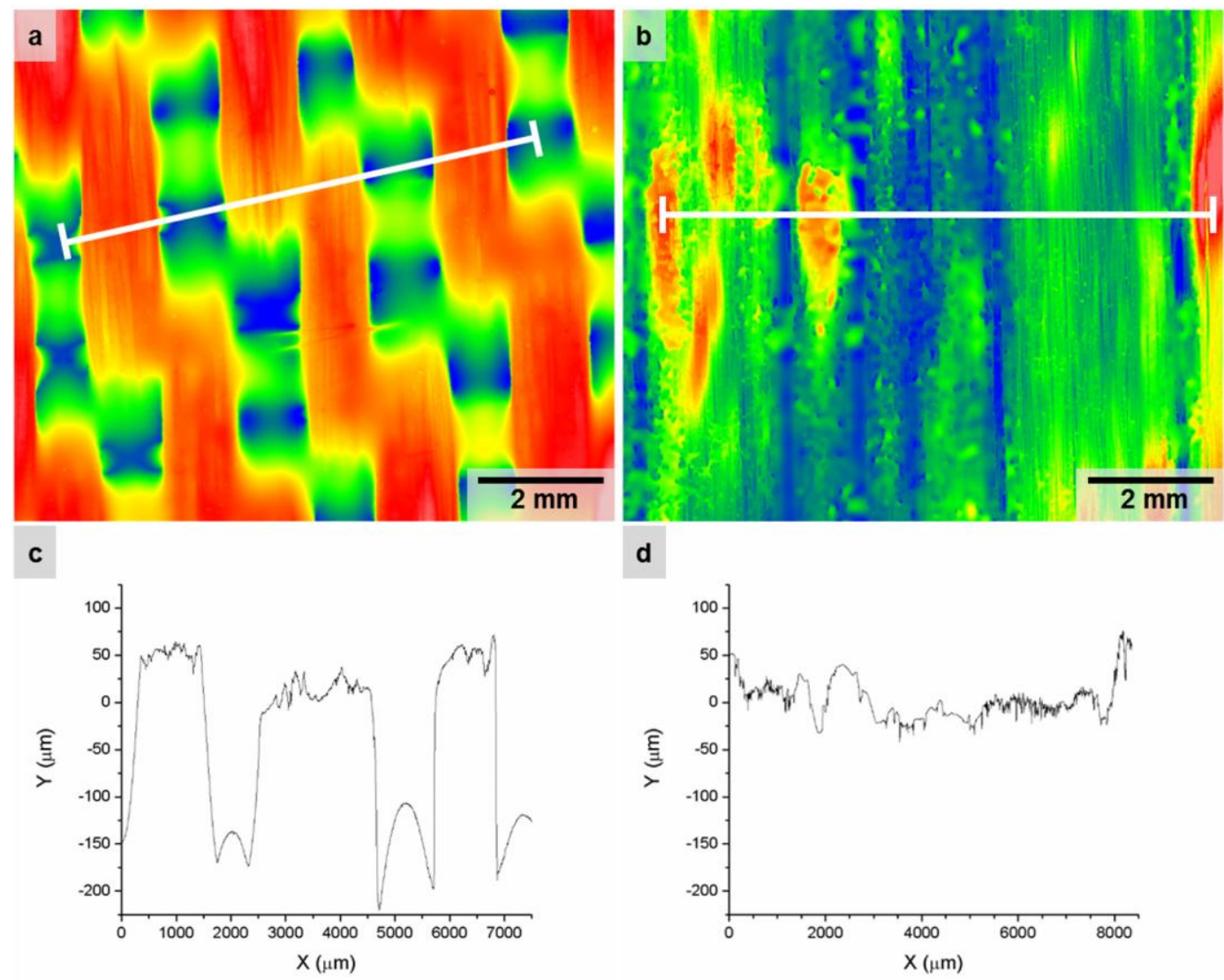

d

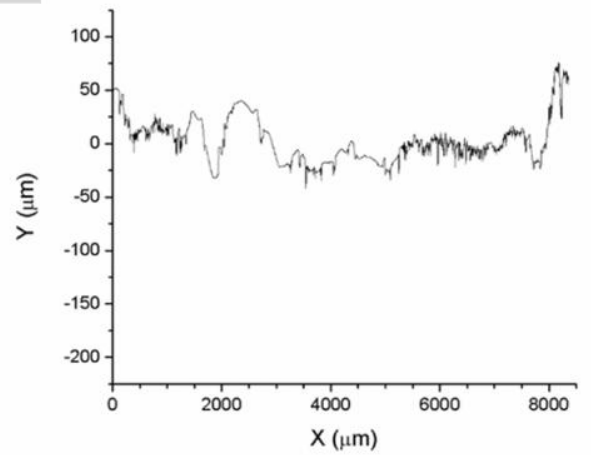




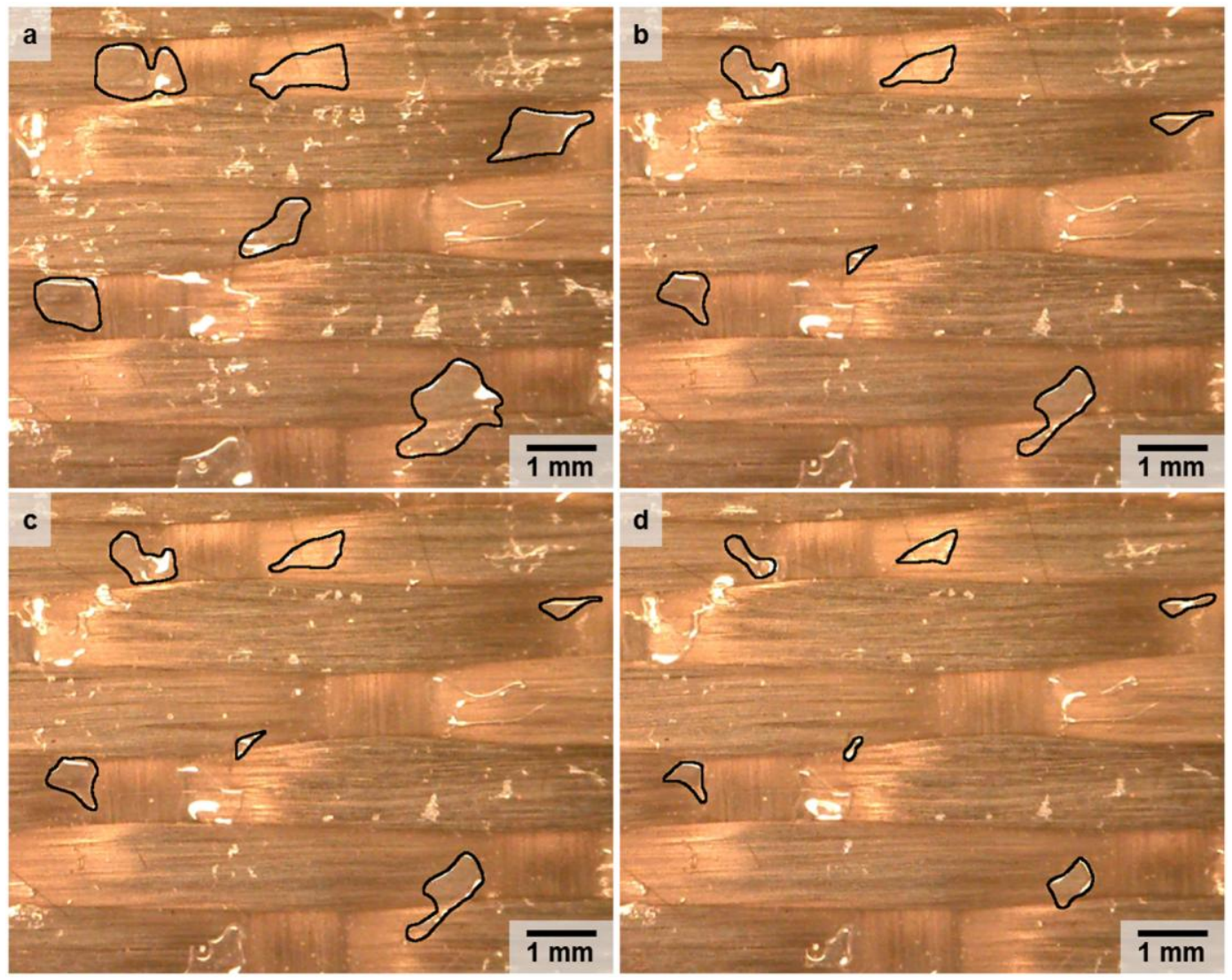




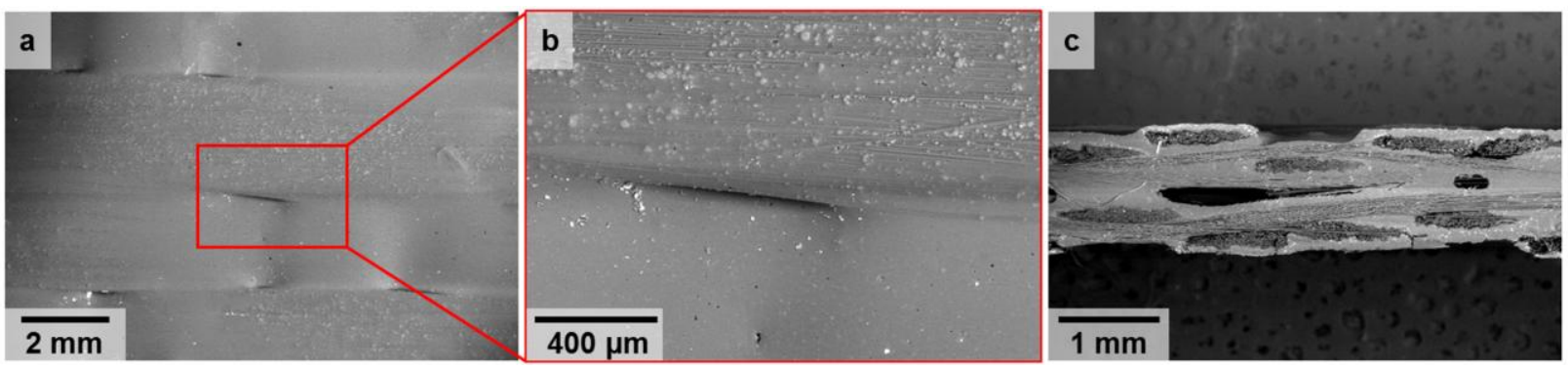




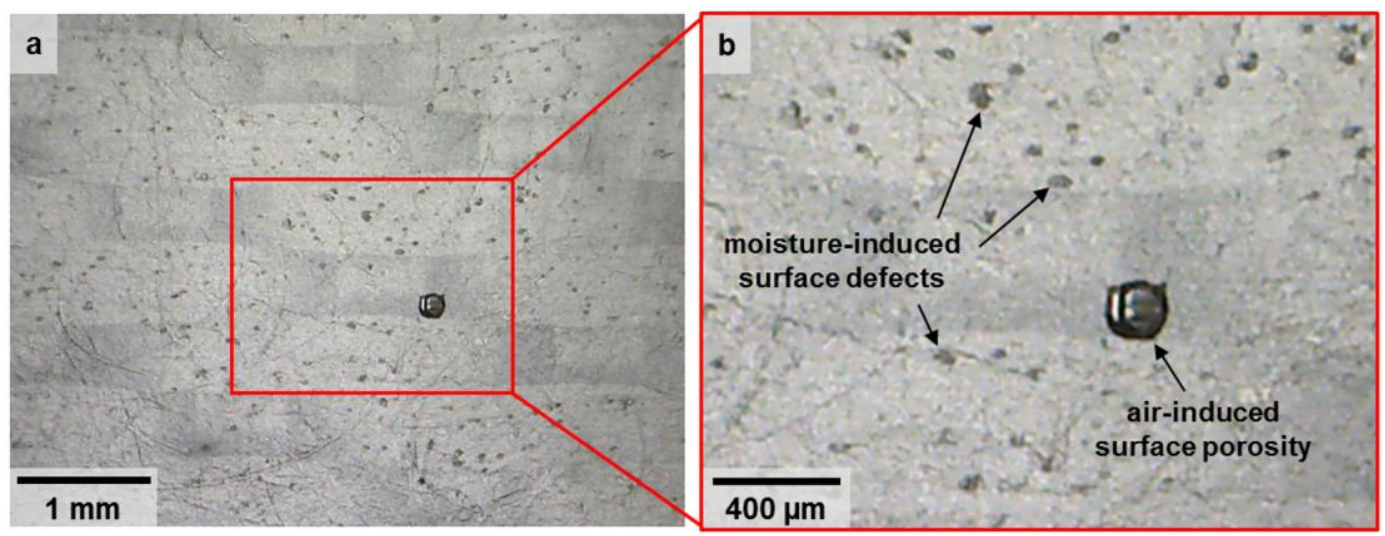


a

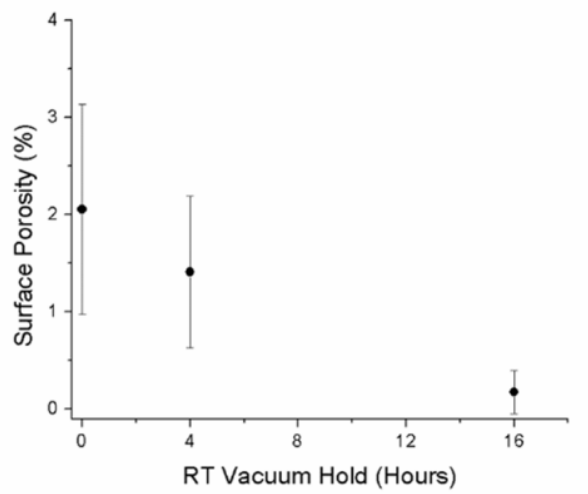

c

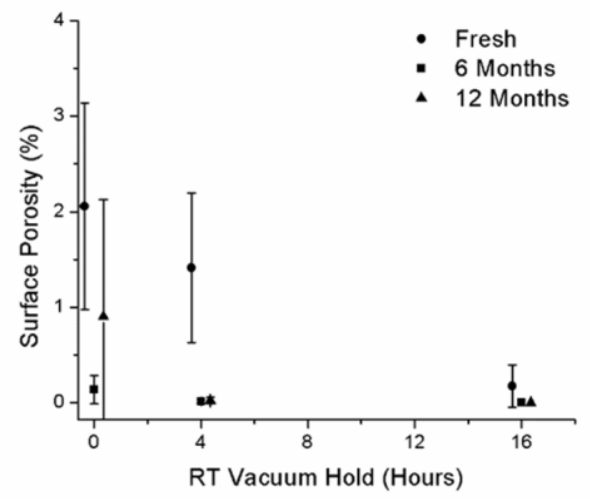

b

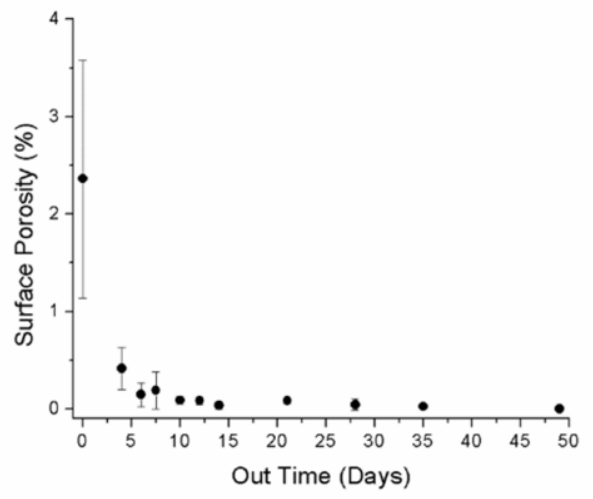

d

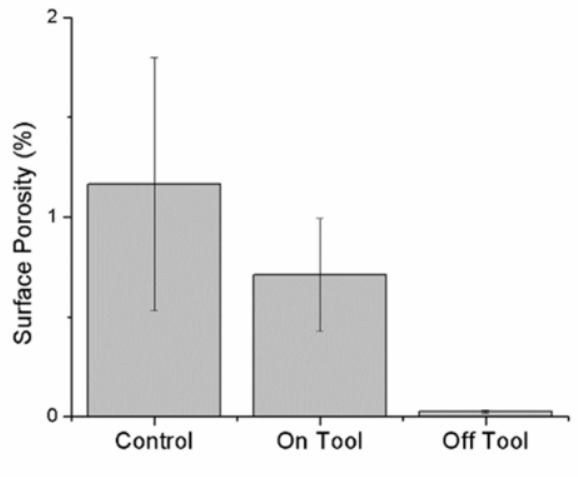




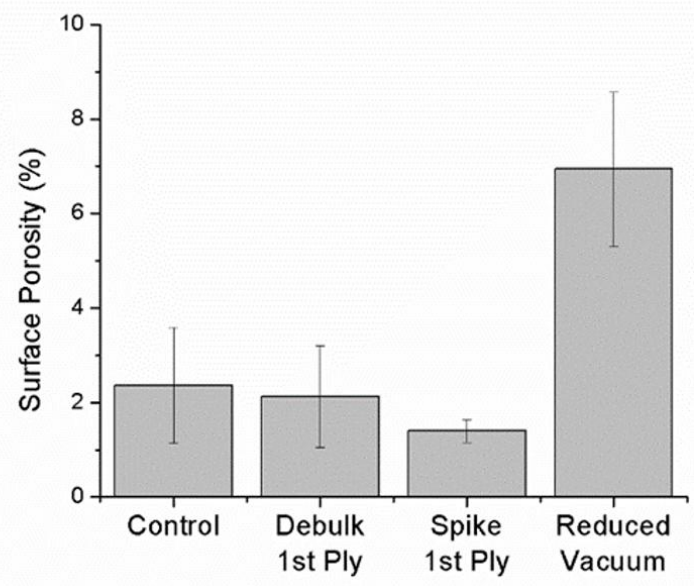

